\title{
Problem gambling and family violence in the Asian context: a review
}

\author{
Brittany Keen*, Dylan Pickering, Martin Wieczorek and Alex Blaszczynski
}

\author{
${ }^{*}$ Correspondence: \\ Brittany.keen@sydney.edu.au \\ Responsible Gambling \\ Research Group, Faculty \\ of Science, School \\ of Psychology, Sydney \\ University, 110 Darlington Rd, \\ Darlington 2008, Australia
}

\begin{abstract}
Background: Few empirical studies have specifically evaluated the causal connection between problem gambling and domestic or family violence within East and SouthEast Asian populations. Evidence from qualitative and quantitative studies suggests that at the very least, an association exists. Lifetime prevalence of intimate partner violence has been drawn from various Asian communities, and ranges from 15.4 to $61.1 \%$, which is generally higher than in western countries such as US, UK and Australia (17-26\%). Problem gambling rates are similarly higher in certain Asian countries (3.8-6\%), when compared to the international average (2.3\%). The aim of this review was to evaluate the available literature on problem gambling and family violence in East and South-East Asian populations, and to highlight any consistencies between the two phenomena. The authors conducted an online literature search using the terms: "Asia", "gambling", "domestic violence", "family violence", and "abuse"; and accessed physical libraries for literature not available online.
\end{abstract}

Discussion: As evidenced by qualitative and quantitative studies, it is reasonable to suggest that a link does indeed exist between family violence and problem gambling in certain Asian communities. The authors argue that there may be specific cultural factors that underpin the acceptance, maintenance, and under reporting of both problem gambling and family violence in some Asian communities. Such cultural factors include: patriarchal family systems, low help seeking and under reporting, the impact of collectivist culture on gambling normalisation, and immigration and acculturation stresses.

Summary: This paper concludes East and South-East Asian communities display high rates of problem gambling and family violence. At the very least, there is some evidence to suggest a relationship between these two issues; however, immigration stresses and cultural beliefs may mediate this effect amongst migrant samples. The current state of research in this area is incomplete and requires rigorous methodological inquiry. Given strong preliminary evidence of a problem gambling-family violence link, it is recommended that future research endeavours focus on accurately assessing a causal relationship between problem gambling and family violence. Further research should be longitudinal, carried out in Asian countries, and use local resident populations to consider whether or not underlying cultural traditions and beliefs engender or contribute to problem gambling and family violence. These findings have implications for the field of problem gambling and family violence by informing prevention and treatment strategies and services within the public health and welfare sectors.

Keywords: Problem gambling, Gambling, Domestic violence, Family violence, Asia, Cultural

\section{望 Springer}

(c) 2015 Keen et al. This article is distributed under the terms of the Creative Commons Attribution 4.0 International License (http:// creativecommons.org/licenses/by/4.0/), which permits unrestricted use, distribution, and reproduction in any medium, provided you give appropriate credit to the original author(s) and the source, provide a link to the Creative Commons license, and indicate if changes were made. 


\section{Background}

\section{The scope of this review}

High rates of problem gambling and family violence exist in many Asian communities (Agbayani-Siewert and Flanagan 2000; Alegría et al. 2009; Bennett et al. 2011; Blaszczynski et al. 1998; Chan 2005; Lee 2007; Liao 2008; Nguyen and Yoshioka 2006; Thomas and Yamine 2000; Williams et al. 2012; Xu et al. 2005; Yick 2000; Yoshihama 1999). International empirical and qualitative research has suggested that a link may exist between the two behaviours (Afifi et al. 2010; Bellringer et al. 2008; Bland et al. 1993; Breen et al. 2013; Fehringer and Hindin 2014; Liao 2008; Lorenz and Shuttlesworth 1983). There are very few studies that specifically evaluate the association between problem gambling and domestic violence within Asian populations. Of those that do, there lacks robust quantitative data to infer that a causal relationship exists. However, evidence from qualitative studies suggests that this may indeed be the case, and that at the very least, further research is warranted in the area. The following scoping review provides an overview of the prevalence of problem gambling and domestic violence in Asian communities. It critically analyses the current literature and examines evidence that may support a link between the two issues. Culturally-specific norms and belief systems are examined as means for the propagation of domestic violence and problem gambling, paying particular attention to gender roles, ideals of masculinity, and settlement issues in migrants. The review highlights the importance of immigration and acculturation stresses on the development and maintenance of problem gambling, relationship breakdown among gamblers, the under reporting of violence, and low help seeking among problem gamblers. The problematic issue of causality within the current literature is clearly indicated. In completing this review we acknowledge, (1) the extensive cultural diversity of the Asian continent, (2) that as a whole, Asian communities do not tolerate domestic violence, and (3) the limitations associated with a review conducted by Western, Englishspeaking authors.

\section{Search method}

The following review is intended to provide a scope of the available literature regarding domestic violence and problem gambling in Asian communities, and is not intended to be a systematic review of all published work on the issue. The authors conducted a web-based search of the literature using varying combinations of the keywords: "Asia", "gambling", "problem", "domestic violence", "family violence" and "abuse". Keywords were searched through the Sydney University library web page, powered by Serial Solutions Summon ${ }^{\mathrm{TM}}$. The University of Sydney's Fisher Library was also accessed in order to obtain physical copies of articles and books not accessible online.

\section{Acknowledging the diversity of the Asian continent}

The Asian continent consists of 55 sovereign states and territories, each with their own unique set of cultural norms, traditions, and values. It is vital to stress that the authors' intentions were not to generalise the findings of this review to all Asian countries, provinces, and cultural groups. Rather, the intention was to investigate and highlight the trends (if any) that appear within the literature with regards to problem gambling and family violence in Asian communities. In doing so, the authors have narrowed the scope 
of this review to focus primarily on East and South-East Asian communities, with some mention of Asian-Pacific countries close to Australia.

\section{Discussion}

\section{A working definition of family and domestic violence}

Defining domestic violence (DV) is difficult. It is variably defined as violence between intimate couples only, described as interpersonal violence (IPV); (Lee 2007; Liao 2008; Raj and Silverman 2002) or abuse toward children or other family members (family violence) (Dowling et al. 2014; Raylu and Oei 2009; Suomi et al. 2013; Tse 2007). Furthermore, although most definitions of DV comprise non-physical acts of aggression (Muelleman et al. 2002; Suomi et al. 2013), there are many studies that operationalise it in purely physical terms (Afifi et al. 2010; Liao 2008). There are also conflicting views as to whether DV by nature is mono-directional (i.e., male $>$ female or female $>$ male) (Taft et al. 2001) or reciprocal (i.e., male $><$ female) (Suomi et al. 2013). The current review adopts the following Australian Bureau of Statistics (ABS) (2013) comprehensive definition of family and domestic violence that takes into account various expressions of violence.

"Family and domestic violence covers a wide range of abusive behaviours committed in the context of intimate relationships such as those involving family members, children, partners, ex-partners, or caregivers. It can include many types of behaviour or threats, including: physical violence, sexual abuse, emotional abuse, verbal abuse and intimidation, economic and social deprivation, damage of personal property and abuse of power." (ABS 2013)

For the purpose of this review, the term 'family violence' (FV) will be used, as it more accurately represents disruption to the entire family unit. Although this definition is wide in scope, it is relevant to note that the majority of studies reviewed have adopted a more narrow focus by examining physical violence between intimate partners. Accordingly, the current review is primarily limited to research on intimate partner violence (IPV).

\section{Prevalence of family violence in various Asian communities}

The prevalence of family violence in various Asian communities varies significantly between different countries, regions, samples (i.e., Asian immigrants living in foreign countries vs. country of origin), and cultural sub-groupings. Lifetime prevalence of IPV for Asian sub-populations has been estimated for the following international and Asian jurisdictions. IPV rates in country of origin: China (15.7-43\%) (Chan 2005; Xu et al. 2005), Indonesia (43\%) (Bennett et al. 2011), Japan (15.4\%) (World Health Organisation [WHO] 2005), Philippines (17.9\%) (National Statistics Office 2008), Thailand (41.147.4\%) (WHO 2005), Vietnam (34.4\%) (United Nations 2010); and IPV among Asian communities living abroad: Chinese (18-45.2\%) (Liao 2008; Yick 2000), Filipino (31.4\%) (Agbayani-Siewert and Flanagan 2000), Japanese (61.1\%) (Yoshihama 1999), Korean (29.4\%) (Lee 2007), and Vietnamese (31\%) (Nguyen and Yoshioka 2006) immigrants living in the US. Generally speaking, these rates are higher than comparative national rates

observed in western countries such as Australia (17\%), US (25\%) and UK (26\%) (ABS 
2012; Mirrlees-Black 1999; Tjaden and Thoennes 2000). It is relevant to note that the reported estimate figures pertaining to Asian immigrants tend to be slightly higher than those living in their country of origin; consequently, it is possible that the elevated rates of violence in "Asian communities" abroad may be at least partly attributable to transmigrational stresses. Nevertheless, it seems plausible that cultural differences may also play a role, given the similarly high rates of violence in local Asian jurisdictions (Bennett et al. 2011; Chan 2005; Xu et al. 2005). Notably, the aforementioned prevalence statistics pertain to IPV perpetrated against women, given their significantly higher rates of victimisation compared to males. The exception is studies by Liao (2008) and Yick (2000), whose data reflect total IPV victimisation across genders, and Nguyen and Yoshioka (2006) who measured male-perpetrated IPV.

Of concern, it is possible that reported violence rates are much lower than in reality, due to high levels of under reporting by victims, which is also evidenced in Asian communities (Aujla 2013; Ross 2014; Shankar et al. 2013). For example, survey data from the Project AWARE Study conducted in Washington DC (McDonnell and Abdulla 2001) revealed that $44.8 \%$ of female respondents living in the US from various Asian backgrounds did "nothing" to protect themselves, and 32.1\% "kept quiet" about the abuse that they, or women they knew, experienced. The same study also found that only $15.7 \%$ of victims or their friends reported calling the police, while 9\% sought help from an agency. These figures highlight the presence of potential cultural barriers to reporting and help seeking in migrant Asian communities. The potential role of cultural values and traditions in propagating domestic violence is explored in more detail later in this review.

\section{Gambling and problem gambling in Asia}

Gambling is a popular and acceptable social activity among many Asian communities around the world (Hobson 1995). Indeed, 'social gambling' is an integral part of the entertainment that occurs during many Asian countries' festive periods (e.g., Chinese New Year) and special events such as birthdays and weddings (Oei and Raylu 2010). Interestingly, overall rates of gambling participation in Asian communities appear to be somewhat lower than those among Western cultures (40-65\% vs. 60-78\%, respectively) (Blaszczynski et al. 1998; Fong Ka-Chio and Ozorio 2003; Home Affairs Bureau [HAB] 2012; Hing et al. 2014; Kessler et al. 2008; National Council on Problem Gambling [NCPG] 2012; Productivity Commission 2010). Though, such findings may be attributable to Asian respondents not regarding certain activities as gambling if played for entertainment/festive reasons (e.g., mah-jong, lotteries) (Blaszczynski et al. 1998). Similar to other cultures, the majority of Asian gamblers are able to gamble responsibly, in such a way that does not cause them or others harm; however, a certain number gamble beyond their affordable limits.

In a recent meta-review, Williams et al. (2012) reported that the highest rates of problem gambling in the world were found in Asian countries, with the exception of South Korea. Standardised averages across a decade (1991-2011) show a problem gambling prevalence of 5.6\% in Hong Kong, 6\% in Macau, and 3.8\% in Singapore; compared to the international average of $2.3 \%$ (Williams et al. 2012). Furthermore, Asian groups living in Western countries have demonstrated relatively higher rates of problem gambling than 
the host society. Studies with mostly Chinese-speaking, Asian immigrant samples, have reported problem gambling rates 1.5-5 times higher than those of the local population (Alegría et al. 2009; Blaszczynski et al. 1998; Thomas and Yamine 2000). Additionally, it has been shown that Asian people are often reluctant to report gambling problems, for fear of losing respect from their family, friends, and community members (Loo et al. 2008).

\section{Gambling forms}

Among the most common forms of gambling in East and South-East Asian provinces are Mark Six lottery (Hong Kong), "flower cards" (Korea), social forms of gambling such as playing mah-jong (Eastern and South Eastern Asia) or poker with friends or relatives, followed by horse racing and casino games (Home Affairs Bureau [HAB] 2012; Park et al. 2010). Evidently, games of some skill appear to be more popular among Asian gamblers than are games purely based on chance (e.g. electronic gaming machines and lottery) (Home Affairs Bureau [HAB] 2012; Park et al. 2010).

Preferences for the mode of gambling among Asian groups may also be influenced by cultural beliefs and values that are maintained through inter-generational effects. Dice and card games, for instance, have been a part of oriental culture for centuries; thus potentially explaining the attraction held by Asian gamblers toward casino tables (Raylu and Oei 2004). Accordingly, one study conducted in Sydney, Australia, found that casino gambling is most popular among Chinese, Vietnamese, and Korean participants compared to other ethnic groups (GAMECS project 1999). Another revealed that more than $50 \%$ of gambling expenditure in the Sydney Chinese community was spent at casinos (McMillen et al. 2004).

\section{Exploring the link between family violence and problem gambling}

Numerous empirical studies have demonstrated correlational links between FV and problem gambling (Afifi et al. 2010; Bland et al. 1993; Liao 2008; Lorenz and Shuttlesworth 1983). Furthermore, multiple lines of evidence suggest that this relationship holds in several non-western cultural groups, including Indigenous Australians (Breen et al. 2013), Phillipinos (Fehringer and Hindin 2014), Pacific Islanders (Bellringer et al. 2008), and Chinese-Americans (Liao 2008). Generally speaking, the reported rates of FV perpetration among problem gamblers range from 16\% (Afifi et al. 2010) to 56\% (Korman et al. 2008), whereas estimates of FV victimisation among problem gamblers fall between 22\% (Afifi et al. 2010) and 68.6\% (Echeburua et al. 2011). The same studies found that pathological gamblers were 6-28 times more likely to commit acts of FV than non-gamblers (Afifi et al. 2010; Liao 2008).

Unfortunately, very few of these studies distinguish between FV of varying natures or severity. The exceptions being Afifi et al. (2010) who differentiate minor assaults (e.g., push, grab, etc.) from major ones (choked, punched, etc.); and Bellringer et al. (2008) who distinguished between verbal and physical acts of violence. Typically, there appears to be a paucity of research identifying the prevalence of FV that is at a level of severity sufficient to warrant external intervention. Such cases represent a quantifiable cost to society and therefore are of particular interest to policy makers. Further, the findings that problem gambling is associated with both perpetration and victimization in IPV, 
suggests a complex causal relationship. Theoretically, two logical paths of causality may be identified. Firstly, due to large losses suffered, problem gamblers may be the perpetrator or victim of $\mathrm{FV}$, in that they may perpetrate aggressive acts in order to vent frustration and stresses associated with gambling losses, or that they are violently targeted by family members disgruntled by their gambling behaviour. Secondly, in the reverse direction, gambling may function as a refuge or distraction for problem gamblers from an existing turbulent family environment (Afifi et al. 2010; Echeburua et al. 2011; Fehringer and Hindin 2014). Longitudinal studies may elucidate the circumstances under which problem gambling is likely to lead to family violence, and conversely, the mechanisms by which exposure to family violence increases the risk for problem gambling.

\section{Family violence and problem gambling in the Asian context}

Few empirical studies have directly examined the link between family violence and problem gambling in Asian communities. However, given the relatively high levels of both family violence and problem gambling found in Asian populations independently, there is good reason to believe that a robust relationship exists between the two, similar to the one evidenced among various other cultural groupings (Bellringer et al. 2008; Breen et al. 2013; Fehringer and Hindin 2014; Liao 2008). Furthermore, it could reasonably be argued that specific cultural norms such as rigid, hierarchical family structures found in many Asian cultures may also make the family violence-problem gambling link more pronounced. To date, a dearth of empirical studies have explored such a link, though a number of studies highlight the link between problem gambling and relationship breakdown in the Asian context.

\section{Country of origin}

Two studies from Korea have found higher rates of relationship breakdowns, separation and divorce among populations of problem and pathological gamblers. For instance, Park et al. (2010) reported on a sample of 5,333 South Korean residents, 43 of who were pathological gamblers. The authors found that pathological gamblers were around three times more likely to be separated/divorced or widowed, and were 2.8 times more likely to suffer from a psychiatric disorder. Similarly, Chun et al. (2011) reported that among a sample of 12 South Korean problem gamblers, all had experienced disrupted relationships with family and friends, and $75 \%$ had experienced divorces or separations. Consistent with this finding, a small qualitative analysis from Macau involving four problem gamblers reported relationship problems and breakdowns as one of the adverse affects associated with gambling (Fong Ka-Chio and Ozorio 2003). At least one study from Hong Kong highlights the probable bi-directional nature of the link between family turmoil and gambling, with Home Affairs Bureau (HAB) (2012) reporting family arguments as a commonly cited trigger for gambling, among a sample of 10 problem and pathological gamblers. While it is unclear whether the high rates of relationship breakdown among gamblers is due to $\mathrm{FV}$, this data suggests that gambling can detrimentally affect one's interpersonal relationships, and increase turbulence within intimate relationships.

For some, violence may indeed be both a reaction to and part of gambling-related relationship problems, albeit an extreme one. A number of studies provide convergent evidence in support of the gambling-family violence link in the Asian context. For instance, 
$\mathrm{Xu}$ et al. (2005) found that among a sample of 600 Chinese women attending a gynaecological clinic in Fuzhou, China, frequent quarrelling was the strongest predictor of IPV in the last 12 months, and critically, that women were at a greater risk of IPV if they refused or were refused money by their partner. This strongly suggested that financial arguments were a significant contributing factor to those IPV cases. These findings align with those of Gallin (1997) who found that local Taiwanese women reported being beaten for refusing to hand over money for the purposes of gambling and alcohol. The abuse was often seen as a justified means of preserving hierarchical family structures. These studies suggest that financial arguments attributable to gambling may precipitate family violence in at least a proportion of cases, and furthermore, highlight the roles that patriarchal family structures may play in the gambling-violence link.

\section{Migrant communities}

Three studies have specifically examined the relationship between gambling and family violence among Asian communities living in western countries. Petry et al. (2003) collected data from 96 South-East Asian refugees living in the US and found that $59 \%$ of the sample met criteria for problem gambling, and that $39 \%$ of those were divorced or separated, compared to only $11 \%$ of the non-problem gamblers in the sample. Similarly, Liao (2008) reported on a sample of 31 Chinese community members in San Francisco, and found that partners of problem gamblers were 27.5 times more likely to experience any intimate partner violence than those whose partners were not problem gamblers. Tse (2007) conducted a qualitative analysis of family violence amongst 56 Chinese, South Asian and South-East Asian immigrants living in New Zealand, and found that gambling was amongst the reported catalysts for violence. Other stressors associated with migration such as employment concerns and financial hardship were also reported.

The heightened levels of family violence attributable to gambling, especially that reported by Liao (2008), suggests that the patriarchal family structures prevalent in certain Asian cultures may indeed exacerbate the gambling-family violence link. Although, as was mentioned above, because the majority of the available data is subjective, and correlational, it should be noted that the strengthened association between gambling and family violence among these migrant communities might be attributable to other stressors associated with the migrant experience.

\section{Exploring cultural factors}

The above analysis strongly suggests the possibility that certain cultural values that are present to varying degrees in some East and South-East Asian cultures, may have a part to play in exacerbating the link between problem gambling and family violence. The following section further explores: (1) the manifestation of patriarchal values and how they may relate to family violence, and (2) cultural factors which may confer increased risk of problem gambling.

\section{Patriarchal values and family violence in the Asian context}

It is argued by some authors that cultural factors play a major role in the acceptance, maintenance, and underreporting of family violence in certain Asian communities that share particular cultural norms and/or beliefs (Bennett et al. 2011; Tse 2007; Xu et al. 2005; 
Yoshihama 2000; Yoshioka and Dang 2000). For instance, pervasive patriarchal belief systems that engender male dominance, authority, and economic control may promote submission in women, and underlie the controlling nature of family violence (Dempsey and Day 2011; Hunnicutt 2009). The pervasiveness of such patriarchal belief systems may be indirectly evidenced by markedly skewed male/female gender ratios at birth in countries such as China (118/100; 2009), South Korea (106.7/100; 2010), and Vietnam (111.2/100; 2010) (Guilmoto 2012). These gender statistics at least in part, reflect practices of prenatal sex selection, abortion of female fetuses, and neglect and abandonment of female children (Hesketh and Xing 2006; Lee 2015; Niaz and Hassan 2006).

\section{Family violence and patriarchal values in Asian migrant communities}

For Asian migrants, settlement issues including financial strain caused by unemployment, isolation, and lack of acculturation may play a role in the development of both FV and PG (Lee 2007; Tran 1999; Tse 2007). Though, the available research suggests that at the very least, cultural factors appear to also play a role in the problem gambling-family violence link. A number of qualitative studies have evidenced the enduring influence of patriarchal values in Asian migrants' experience of family violence. For instance, Yoshihama (2000) found that $71 \%$ of Japanese women living in an abusive relationship in Los Angeles believed that the experience of their partners' violence was influenced by their Japanese culture.

A number of culturally specific beliefs associated with the under-reporting of family violence, were identified in qualitative studies evaluating attitudes of family violence victims in various Asian communities.

(a) Conflict avoidance facilitated by the suppression of emotion and self-blame.

"... I wouldn't confront the situation. I would try to solve the problem in my

head. I basically thought the problem was me..." (3rd generation Japanese in US; Yoshihama 2000, p. 215)

(b)Endurance to tolerate abuse in a marriage, uncomplaining and with grace.

"Your body belongs to him until he dies... he can do anything he wants to dothere is no concept of marital rape." (Asian woman in New Zealand; Tse 2007, p. 176)

(c) Acceptance of male domination women should be submissive, subservient, and subordinate.

"The husband is like God" ... "a man should always control his wife... he should never accept different opinions from his wife".. "Women are brought up to service men, to be obedient, to be submissive." (Asian immigrant women in New Zealand; Tse 2007, p. 177)

(d) Collective family welfare the family unit should remain intact regardless of circumstance.

"...When you get married, you don't leave. You don't divorce, you don't do anything. You just put up with it..." (3rd generation Japanese in US; Yoshihama 2000, p. 217) 
(e) Aversion to help seeking problems are kept internal so to avoid shame.

"Family violence is a private matter... Women can't make complaints against their husband... We need to hide things, thinking what will happen if anybody knows." (Asian immigrant women in New Zealand; Tse 2007, p. 183)

There is evidence that greater exposure to local norms and relatedly, acculturation, may allow female Asian migrants to re-evaluate their culture-specific roles and behaviours imposed during their upbringing. For instance, Yoshihama (2002) conducted a study that compared Japanese-American women who were born in the US with those who were born in Japan and migrated to the US. The study found that there were significantly higher rates of help seeking ( 82.8 vs. $43.3 \%)$ and confrontation to abusive partners ( 86 vs. $68 \%$ ) by those born in the US compared to those born in Japan (Yoshihama 2002). Such behaviours are indicative of active responses to family violence, which are inconsistent with conservative values promoting passivity. Consequently it appears that perhaps US born Japanese women were less constrained by the cultural expectations and traditions of their ethnic background than those born in Japan. Similarly, Lee (2007) collected survey data from 136 Korean women and conducted multiple regression analyses to find a positive association between low levels of acculturation among Korean-Americans, and male-perpetrated psychological assault. Further, Korean immigrant women who were part of more traditional families, experienced a higher level of violence when compared to those in less traditional families (Lee 2007). Also, Yick and Agbayani-Siewiert (1997) found that the longer Chinese residents lived in the USA, the more likely they were to disagree that violence was justified when a spouse does not obey his/her partner. However, this study employed a small telephone survey of only 31 respondents, consequently the reliability of their conclusions is questionable. Additionally, it should be noted that although acculturation significantly predicted physical violence in Yick's (2000) study, it was not significantly associated with physical violence or injury in Lee's (2007) study. This indicates that perhaps violence results as a consequence of stressors of the acculturation and migration process, rather than from low levels of acculturation itself.

As suggested above, difficulty adjusting to new gender roles may help to explain some of the violence experienced by Asian immigrants living in Western countries. Tran (1999) interviewed Vietnamese service workers and detailed circumstances in which Vietnamese men living in Australia are faced with feelings of disempowerment following migration. Upon migration to Australia, Vietnamese women often welcome newly acquired freedom and equal rights to men. However, some husbands find this demoralising or threatening, interpreting it to ultimately lead to their obsolescence in the family. This phenomenon has been termed the 'backlash' hypothesis, where the fall from authority to equality can be seen as a loss of control, and therefore, the pursuit to regain control can result in family violence (Tran 1999; Whaley 2001; Yllo 1983).

Tse (2007) depicts a similar narrative, using qualitative data from 56 Asian women who migrated to New Zealand. Focus groups revealed that immigrant wives aspired to adopt the host country's culture and attain paid work. However, husbands perceived this as a loss of control over their wives, and in many cases husbands became "more 
oppressive" (pg. 178) than they were in their home country. This was described as clinging to traditional values, resulting from feelings of uncertainty in a new environment. Consequently, husbands used violence as a means to reinforce social customs and practices when they felt they were losing control over their wives. Moreover, husbands were frequently reported to have threatened to send their wives back to their home country if they did not comply with their demands (Tse 2007). Such intimidation represents a unique threat to immigrants experiencing family violence.

It should also be noted that accepted definitions of IPV among Asian communities appear to depend greatly on contextual factors. For example, in some Asian communities, declarations of rape are seen to be less legitimate or sometimes even illegitimate if they occur within the bounds of a marriage or intimate relationship (Tse 2007; National Asian Women's Health Organization 2002 as cited in Yoshihama and Dabby 2009). Similarly, around one-third of the 507 Asian-American participants surveyed agreed that husband-perpetrated violence was justified if a wife was unfaithful (36.2\%), or nagged her husband too much (30.8\%) (Yoshioka et al. 2001). Furthermore, while most of the 440 Vietnamese-American phone survey respondents in Bui's (2006) study believed IPV to be a problem in the Vietnamese community, only two in five considered it to be "serious" or "very serious". Interestingly, under half of the sample (46.8\%) said they knew that perpetrators of FV could be prosecuted in a criminal court. Indicating a clear lack of knowledge and education in the area of family violence.

In short, while it appears that persistent traditional values and customs play a role in the perceptions of violence in certain Asian migrant communities, it should be noted that rigorous, empirical research is still scarce in this area, with many of the studies reported relying on subjective accounts of a small number of violence victims or service workers. Notwithstanding, larger-scale quantitative telephone surveys appear to support claims made by smaller qualitative studies.

\section{Cultural factors and problem gambling in Asian communities}

The variance and rates of gambling and problem gambling among Asian populations, both local and migrant, owes considerable explanation to the influence of cultural factors (Oei and Raylu 2007). Reviewing this topic, Raylu and Oei (2004) argue that culture provides the framework through which individuals attribute meaning to gambling and shape how risk is managed, such valuations being passed down historically from one generation to the next. Accordingly, the authors identified three core interrelated variables that contribute significantly to the development and maintenance of problem gambling in both local and migrant Asian communities, including:

\section{Cultural values and beliefs}

Perhaps the most observable way in which cultural beliefs and values manifest is within the family structure. Patriarchal family systems, such as those valued in most Asian cultures, are more likely to have a stronger modeling-impact of parental gambling. For example, children are more likely to gamble if the head of their family (the father) gambles, because their culture requires them to respect and obey him. Raylu and Oei (2004) also suggest that collectivist cultures may experience greater influence on gambling behaviour among its members, and therefore, stronger impacts 
of modeling behaviour. Consequently, compared to individualistic cultures such as in the West, members of collectivist Asian cultures may be more or less inclined to gamble (and gamble to excess) if members of their cultural group do.

\section{Issues with acculturation/immigration}

Immigration stressors including difficulties with acculturation may play a mediating role in the development of gambling problems. However, the precise mechanisms by which acculturation per se affects problem gambling are not entirely clear. Raylu and Oei (2004) describe two theories regarding this association. The first is that less acculturation leads to more stressors including psychological, financial, and unemployment problems, and that these stressors increase one's risk of developing problem gambling (Raylu and Oei 2004; Tse 2007). For example, adapting to life in a new country carries an increased risk of experiencing unique hardships related to minority status and acculturation, specifically in familial (e.g., intergenerational differences), social (e.g., perceived discrimination), and economic (e.g., poor finances) domains (Berry 1997; Cervantes and Cordova 2011). Furthermore, the process of immigration is commonly associated with feelings of boredom, isolation, and loneliness that can lead to mental illness (Jamil et al. 2007; Roche and Kuperminc 2012; Trevorrow and Moore 1998). These constructs are also frequently reported as motivations for gambling among problem gamblers (Blaszczynski and Nower 2002; Hallebone 1999), thus suggesting that immigrants may be at a higher risk of developing gambling problems. Conversely, successful acculturation to a host country that endorses gambling behaviour may act to normalize betting and therefore increase one's risk of developing gambling problems (Raylu and Oei 2004).

Data from the Victorian Casino and Gambling Authority (VCGA) (1999) appears to support the former hypothesis. In addition to feelings of loneliness and boredom, the effects of migration were cited as some of the most common reasons for gambling by Asian-Australian migrants (Victorian Casino and Gambling Authority [VCGA] 1999). However, acculturation may play a larger role in the maintenance and progression of gambling problems by effecting help seeking behaviours (Atkinson and Gim 1989). Immigrants who are more acculturated to their host country are more likely to adopt the help seeking behaviours of that country (Tata and Leong 1994). Likewise, less acculturated immigrants who have come from a low help seeking background may be less inclined to seek help, even when help is available in the host country. Therefore, it is likely that less acculturated Asian people (from low-help seeking backgrounds) living in the West (higher help seeking behaviours) will be less inclined to pursue assistance when it is needed.

\section{Attitudes toward seeking professional help}

Low help seeking is common among cultures such as the Chinese that prefer coping strategies with high reliance on self-help measures, and family and community support (Cheung 1986; Luk and Bond 1992). Further, collectivist cultures such as those in Asia are more likely to hide gambling problems due to feelings of shame and stigma associated with problem gambling (Scull and Woolcock 2005). As such, gamblers will often be concealed within the family, where the head of the family decides on alternative support and treatment (such as traditional healing and herbal medicine) (Cheung 1993). It is likely that by not seeking help early, and attempting to 
hide gambling problems, or self-manage, that problems may become more severe, increasing the likelihood of a later gambling disorder. Additionally, Asian communities living in Queensland, Australia have reported a lack of culturally appropriate support services for problem gambling, further contributing to the lack of help seeking among this population (Scull and Woolcock 2005).

\section{Acknowledging diversity and limitations}

The Asian continent is vast, and is comprised of a great diversity of countries, peoples, and cultures. To encompass every country belonging to the Asian continent is beyond the scope of the this review and would deviate from its purpose, therefore the focus has been narrowed to literature on East and South-East Asian communities, and Asian immigrants living abroad.

Perhaps the most evident limitation of this review is the authors' Western cultural backgrounds. Language barriers limited the authors from including articles published in languages other than English. This has likely resulted in a bias of studies conducted in or by western countries such as the US, Canada, the UK, Australia and New Zealand, and an overrepresentation of Asian migrant samples rather than Asian residents. Furthermore, because of a lack of exhaustive research in this niche area, few robust conclusions can be drawn, emphasising the need for further studies evaluating the problem gambling-family violence link in Asian populations. Finally, while this review explores the cultural underpinnings that may influence family violence, we emphasise that on the whole, Asian communities do not consider cultural traditions or expectations to be adequate justification for violence and agree that family violence is a problem that should be stopped (Bui 2006; Tse 2007; Yoshioka and Dang 2000).

\section{Recommendations for future research}

It would be helpful if future research endeavours utilised samples of Asian citizens living in Asian countries, rather than Asian migrant communities living abroad. This would provide a more localised and focused view of family violence and problem gambling in Asian countries. While comparative data from Asian migrant communities is a useful reference, the English-speaking literature is typically saturated with studies of this kind, emphasising a need for local research that can be used as a point of reference.

More specifically, future research should focus on studies that directly evaluate the association between family violence and problem gambling in East and South-East Asia, employing methodologies that aim to answer four research questions:

1. Does problem gambling directly lead to family violence?

2. Does family violence exacerbate gambling to the point of a gambling disorder?

3. Are there common underlying cultural traditions and/or belief systems that underpin both problem gambling and family violence and therefore serve as a facilitating third factor (i.e. patriarchal family systems/migration/acculturation)?

4. Are there differences between Asian countries/states/regions in terms of the nature of the problem gambling-family violence relationship and how it is manifested? If so, what are they? 
Methodologies used to test these research questions should also aim to:

1. Collect longitudinal data-this will help to indicate the direction of correlation, i.e. does problem gambling lead to family violence or vice versa.

2. Collect qualitative data that pays particular attention to cultural belief systems around control, masculinity and violence.

3. Collect quantitative data that evaluates the extent to which one type of behaviour causes the other, or is caused by outside cultural factors.

4. Collect qualitative/quantitative data from different Asian areas and compare relevant outcomes between groups.

\section{Summary}

The literature provides a substantial amount of preliminary evidence to suggest that cultural norms and attitudes present in some Asian traditions play a strong role in the development and maintenance of gambling problems, particularly its role in avoidance of help seeking. Similarly, there is evidence that demonstrates traditional patriarchal family systems contribute to the development and concealment of family violence in Asian families. While there is considerable data supporting a link between problem gambling and family violence in Asian communities, a large proportion of the data is subjective, qualitative, and based on very small sample sizes. This is not to say that a link does not exist, rather that sound research, specific to this topic, has not been carried out in order to accurately measure the association and thoroughly investigate its underlying causes. Preliminary evidence suggests that Asian immigrants living abroad should be considered 'at-risk' for both problem gambling and family violence perpetration and victimisation. Likewise, policy makers in Asian jurisdictions should focus on education and awareness campaigns in an effort to increase help seeking for gambling problems and family violence, as well as increasing treatment service availability for both issues.

\section{Abbreviations}

ABS: Australian Bureau of Statistics; DV: domestic violence; FV: family violence; GAMECS: gambling among members of ethnic communities in Sydney survey; HAB: Home Affairs Bureau; IPV: intimate partner violence; NCPG: National Council on Problem Gambling; PG: problem gambling; VCGA: Victorian Casino and Gambling Authority; WHO: World Health Organization.

Authors' contributions

All authors (BK, DP, MW and AB) contributed substantially to (1) the literature review, including searching for, collating and critically analysing research articles and (2) the drafting and revision of the manuscript. All authors read and approved the final manuscript.

Acknowledgements

This was an unfunded project, conducted independently by the authors

Compliance with ethical guidelines

Competing interests

The authors declare that they have no competing interests.

Received: 29 April 2015 Accepted: 9 July 2015

Published online: 25 July 2015 


\section{References}

Affi, T. O., Brownridge, D. A., MacMillan, H., \& Sareen, J. (2010). The relationship of gambling to intimate partner violence and child maltreatment in a nationally representative sample. Journal of Psychiatric Research, 44, 331-337 (2010). doi:10.1016/j.jpsychires.2009.07.010.

Agbayani-Siewert, P., \& Flanagan, A. Y. (2000). Filipino American dating violence: definitions, contextual justifications, and experiences of dating violence. Journal of Human Behavior in the Social Environment, 3(3-4), 115-133 (2000). doi:10.1300/J137v03n03_09.

Alegría, A., Petry, N. M., Hasin, D. S., Liu, S. M., Grant, B. F., \& Blanco, C. (2009). Disordered gambling among racial and ethnic groups in the US: results from the national epidemiologic survey on alcohol and related conditions. CNS Spectrums, 14(3), 132-142.

Atkinson, D. R., \& Gim, R. H. (1989). Asian-American cultural identity and attitudes toward mental health services. Journal of Counseling Psychology, 36(2), 209-212 (1989). doi:10.1037/0022-0167.36.2.209.

Aujla, W. (2013). Voicing challenges: South Asian immigrant women speak out about their experiences of domestic violence and access to services. Masters thesis, Canada: University of Alberta. http://www.academia.edu/2296975/Noicing_ Challenges_South_Asian_Immigrant_Women_Speak_Out_about_their_Experiences_of_Domestic_Violence_and_ Access_to_Services. Accessed 20 Feb 2015.

Australian Bureau of Statistics (ABS) (2012). Personal safety, Australia. Cat no. 4906.0. Canberra. http://www.abs.gov.au/ ausstats/abs@.nsf/mf/4906.0.Accessed 20 Feb 2015.

Australian Bureau of Statistics (ABS) (2013). Directory of family and domestic violence statistics. Cat no. 4533.0. Canberra. http://www.abs.gov.au/ausstats/abs@.nsf/mf/4533.0. Accessed 22 Feb 2015.

Bellringer, M., Abbott, M., Williams, M., \& Gao, W. (2008). Problem gambling: Pacific Islands families longitudinal study. Auckland: Gambling and Addictions Research Centre, Auckland University of Technology. http://www.health.govt.nz/ system/files/documents/pages/pif.pdf. Accessed 10 Feb 2015.

Bennett, L. R., Andajani-Sutjahjo, S., \& Idrus, N. I. (2011). Domestic violence in Nusa Tenggara Barat, Indonesia: Married women's definitions and experiences of violence in the home. The Asia Pacific Journal of Anthropology, 12(2), 146-163 (2011). doi:10.1080/14442213.2010.547514.

Berry, J. W. (1997). Immigration, acculturation, and adaptation. Journal of Applied Psychology, 46(1), 5-34 (1997). doi:10.1111/j.1464-0597.1997.tb01087.x.

Bland, R. C., Newman, S. C., Orn, H., \& Stebelsky, G. (1993). Epidemiology of pathological gambling in Edmonton. Canadian Journal of Psychiatry, 38(2), 108-112.

Blaszczynski, A., Huynh, S., Dumlao, V. J., \& Farrell, E. (1998). Problem gambling within a Chinese speaking community. Journal of Gambling Studies, 14(4), 359-380 (1998). doi:10.1023/A:1023073026236.

Blaszczynski, A., \& Nower, L. (2002). A pathways model of problem and pathological gambling. Addiction, 97(5), 487-499 (2002). doi:10.1046/j.1360-0443.2002.00015.x.

Breen, H., Hing, N., \& Gordon, A. (2013). Indigenous Australian gambling crime and possible interventions: a qualitative study. Asian Journal of Gambling Issues and Public Health, 3(4), 1-16 (2013). doi:10.1186/2195-3007-3-4.

Bui, H. (2006). Perceptions of intimate partner violence and attitudes toward interventions: the role of acculturation. Journal of Ethnicity in Criminal Justice, 3(4), 1-27 (2006). doi:10.1300/J222v03n04_01.

Cervantes, R. C., \& Cordova, D. (2011). Life experiences of Hispanic adolescents: developmental and language considerations in acculturation stress. Journal of Community Psychology, 39(3), 336-352 (2011). doi:10.1002/jcop.20436.

Chan, K. L. (2005). Study on child abuse and spouse battering: report on findings of household survey. A Consultancy Study Commissioned by the SWD of the HKSAR. Hong Kong: Department of Social Work and Social Administration, the University of Hong Kong. http://www.swd.gov.hk/doc/family/Report\%20on\%20findings\%20of\%20Household\%20Survey.pdf. Accessed 10 Jan 2015.

Cheung, F. M. (1986). Psychopathology among Chinese people. In M. H. Bond (Ed.), The psychology of the Chinese people (pp. 171-212). Hong Kong: Oxford University Press.

Cheung, Y. W. (1993). Approaches to ethnicity: clearing roadblocks in the study of ethnicity and substance use. The International Journal of the Addictions, 28(12), 1209-1226.

Chun, J. S., Cho, S., Chung, I. J., \& Kim, S. (2011). Economic and psychosocial impact of problem gambling in South Korea. Asian Journal of Gambling Issues and Public Health, 2(1), 29-38 (2011). doi:10.1007/BF03342123.

Dempsey, B., \& Day, A. (2011). The identification of implicit theories in domestic violence perpetrators. International Journal of Offender Therapy and Comparative Criminology, 55(3), 416-429 (2011). doi:10.1177/0306624X10363448.

Dowling, N. A., Jackson, A. C., Suomi, A., Lavis, T., Thomas, S. A., Patford, J., et al. (2014). Problem gambling and family violence: prevalence and patterns in treatment-seekers. Addictive Behaviours, 39, 1713-1717.

Echeburua, E., Gonzalez-Ortega, I., Corral, P. D., \& Polo-Lopez, R. (2011). Clinical gender differences among adult pathological gamblers seeking treatment. Journal of Gambling Studies, 27(2), 215-227 (2011). doi:10.1007/s10899-010.9205-1.

Fehringer, J. A., \& Hindin, M. J. (2014). 'I get angry if he's always drinking and we have no money': exploring motivations for male and female perpetrated intimate partner violence in the Philippines. Health Care for Women International, 35(4), 476-491 (2014). doi:10.1080/07399332.2013.770003.

Fong Ka-Chio, D., \& Ozorio, B. (2003). Studying gambling participation in Macao: summary of major findings. Institute for the Study of Commercial Gaming. University of Macao. http://www.umac.mo/iscg/Events/Seminar/S1_SummaryReport_ENG.pdf. Accessed 20 Dec 2014.

Gallin, R. S. (1997). Wife abuse in the context of development and change: a Chinese (Taiwanese) case. In N. Visvanathan, L. Duggan, L. Nisonoff, \& N. Wiegersma (Eds.), The women, gender and development reader (pp. 150-155). London: Zed Books.

GAMECS Project (1999). Gambling among members of ethnic communities in Sydney: report on "problem gambling and ethnic communities" (part 3). Sydney: Ethnic Communities' Council of NSW. http://www.olgr.nsw.gov.au/ rr_gamecs_pt_1.asp. Accessed 6 Jan 2015.

Guilmoto, C. Z. (2012). Sex imbalances at birth: Current trends, consequences and policy implications. UNFPA Asia and Pacific Regional Office. http://www.unfpa.org/publications/sex-imbalances-birth. Accessed 18 June 2015.

Hallebone, E. (1999). Women and the new gambling culture in Australia. Loisir Et Societe, 22(1), 101-125. 
Hesketh, T., \& Xing, Z. W. (2006). Abnormal sex ratios in human populations: causes and consequences. Proceedings of the National Academy of Sciences of the United States of America, 103(36), 13271-13275 (2006). doi:10.1073/ pnas.0602203103.

Hing, N., Gainsbury, S., Blaszczynski, A., Wood, R., Lubman, D., \& Russell, A. (2014). Interactive gambling. Melbourne: Gambling Research Australia. http://www.gamblingresearch.org.au/resources/6482d5fa-f068-41e5-921f-facd4f10365e/ interactive+gambling.pdf. Accessed 5 Jan 2015.

Hobson, J. S. P. (1995). Macau: gambling on its future? Tourism Management, 16(3), 237-246.

Home Affairs Bureau (HAB) (2012). The study on Hong Kong people's participations in gambling activities. Hong Kong: The Department of Applied Social Sciences, The Hong Kong Polytechnic University. http://www.hab.gov.hk/file_manager/en/documents/policy_responsibilities/others/gambling_report_2011.pdf. Accessed 10 Jan 2015.

Hunnicutt, G. (2009). Varieties of patriarchy and violence against women: resurrecting "Patriarchy" as a theoretical tool. Violence Against Women, 15(5), 553-573 (2009). doi:10.1177/1077801208331246.

Jamil, H., Nassar-McMillan, S. C., \& Lambert, R. G. (2007). Immigration and attendant psychological sequelae: a comparison of three waves of Iraqi immigrants. American Journal of Orthopsychiatry, 77(2), 199-205 (2007). doi:10.1037/0002-9432.77.2.199.

Kessler, R. C., Hwang, I., LaBrie, R., Petukhova, M., Sampson, N. A., Winters, K. C., \& Shaffer, H. J. (2008). The prevalence and correlates of DSM-IV Pathological Gambling in the National Comorbidity Survey Replication. Psychological Medicine, 38(9), 1351-1360 (2008). doi:10.1017/S0033291708002900.

Korman, L.K., Collins, J., Dutton, D., Dhayananthan, B., Littman-Sharp, N., \& Skinner, W. (2008). Problem gambling and intimate partner violence. Journal of Gambling Studies, 24(1), 13-23 (2008). doi:10.1007/s10899-007-9077-1.

Lee, E. (2007). Domestic violence and risk factors among Korean immigrant women in the United States. Journal of Family Violence, 22(3), 141-149 (2007). doi:10.1007/s10896-007-9063-5.

Lee, M. K. (2015). Progress in the health and nutrition of girls in the Asia-Pacific region. Asia Pacific Journal of Public Health, 27(1), 19-23.

Liao, M. S. (2008). Intimate partner violence within the Chinese community in San Francisco: problem gambling as a risk factor. Journal of Family Violence, 23, 671-678 (2008). doi:10.1007/s10896-008-9190-7.

Loo, J. M. Y., Raylu, N., \& Oei, T. P. S. (2008). Gambling among the Chinese: a comprehensive review. Clinical Psychology Review, 28(7), 1152-1166 (2008). doi:10.1016/j.cpr.2008.04.001.

Lorenz, V. C., \& Shuttlesworth, D. E. (1983). The impact of pathological gambling on the spouse of the gambler. Journal of Community Psychology, 11(1), 67-76 (1983). doi:10.1002/1520-6629(198301)11:1<67::AID-JCOP2290110107>3.0.CO;2-O.

Luk, C. L., \& Bond, M. H. (1992). Chinese lay beliefs about the causes and cures of psychological problems. Journal of Social and Clinical Psychology, 11(2), 140-157 (1992). doi:10.1521/jscp.1992.11.2.140.

McDonnell, K. A., \& Abdulla, S. E. (2001). Project AWARE: research project. Asian/Pacific Islander Domestic Violence Resource Project. Washington, DC. http://www.dvrp.org/. Accessed 6 Dec 2014.

McMillen, J., Marshall, D. Murphy, L., Lorenzen, S., \&Waugh, B. (2004). Help seeking by problem gamblers, friends and families: a focus on gender and cultural groups. Australian National University, Centre for Gambling Research. Australian Capitol Territory. https://digitalcollections.anu.edu.au/bitstream/1885/45186/3/Help-seeking-FINAL-revised. pdf. Accessed 10 Jan 2015.

Mirrlees-Black, C. (1999). Domestic violence: findings from a new British Crime Survey self-completion questionnaire. Home Office Research Study 191. London: Home Office, England. http://webarchive.nationalarchives.gov. uk/20110218135832/http:/rds.homeoffice.gov.uk/rds/pdfs/hors191.pdf. Accessed 10 Jan 2015.

Muelleman, R. L., DenOtter, T., Wadman, M. C., Tran, T. P., \& Anderson, J. (2002). Problem gambling in the partner of the emergency department patient as a risk factor for intimate partner violence. The Journal of Emergency Medicine, 23(3), 307-312.

National Council on Problem Gambling (NCPG) (2012). Report of survey on participation in gambling activities among Singapore residents, 2011. National Council on Problem Gambling. http://www.ncpg.org.sg/en/Pages/Publication. aspx. Accessed 10 Jan 2015.

National Statistics Office (2008). Philippines National Demographic and Health Survey 2008. NSOM and Macro International. http://dhsprogram.com/pubs/pdf/FR224/FR224.pdf. Accessed 15 June 2015.

Nguyen, T. D., \& Yoshioka, M. (2006). Alcoholism level differences between Vietnamese batterers and non-batterers. Journal of Family Violence, 21(6), 401-406 (2006). doi:10.1007/s10896-006-9037-z.

Niaz, U., \& Hassan, S. (2006). Culture and mental health of women in South-East Asia. World Psychiatry, 5(2), $118-12$.

Oei, T. P. S., \& Raylu, N. (2007). Gambling and problem gambling among the Chinese. Research and Community Engagement Division, Queensland Office of Gaming Regulation. Brisbane: Queensland Treasury. https://publications.qld. gov.au/storage/f/2014-06-20T01\%3A31\%3A16.559Z/gambling-and-problem-gambling-among-the-chinese.pdf. Accessed 20 Dec 2014.

Oei, T. P. S., \& Raylu, N. (2010). Gambling behaviours and motivations: a cross-cultural study of Chinese and Caucasians in Australia. International Journal of Social Psychiatry, 56(1), 23-34 (2010). doi:10.1177/0020764008095692.

Park, S., Cho, M. J., Jeon, H. J., Lee, H. W., Bae, J. N., Park, J. I., Sohn, J. H., Lee, Y. R., Lee, J. Y., \& Hong, J. P. (2010). Prevalence, clinical correlations, comorbidities, and suicidal tendencies in pathological Korean gamblers: results from the Korean Epidemiologic Catchment Area Study. Social Psychiatry and Psychiatric Epidemiology, 45(6), 621-629 (2010). doi:10.1007/s00127-009-0102-9.

Petry, N. M., Armentano, C., Kuoch, T., Norinth, T., \& Smith, L. (2003). Gambling participation and problems among South East Asian refugees to the United States. Psychiatric Services, 54(8), 1142-1148 (2003). doi:10.1176/appi.ps.54.8.1142.

Productivity Commission. (2010). Gambling. Inquiry Report. No. 50. Canberra. http://www.pc.gov.au/inquiries/completed/gambling-2009/report. Accessed 20 Dec.

Raj, A., \& Silverman, J. G. (2002). Intimate partner violence against south Asian women in greater Boston. Journal of the American Medical Women's Association, 57(2), 111-114.

Raylu, N., \& Oei, T. P. S. (2004). Role of culture in gambling and problem gambling. Clinical Psychology Review, 23, 1087-1114 (2004). doi:10.1016/j.cpr.2003.09.005. 
Raylu, N., \& Oei, T. P. S. (2009). Factors associated with the severity of gambling problems in a community gambling treatment agency. International Journal of Mental Health Addiction, (7), 124-137 (2009). doi:10.1007/s1 1469-008-9160-6.

Roche, C., \& Kuperminc, G. P. (2012). Acculturative stress and school belonging among Latino youth. Hispanic Journal of Behavioral Sciences, 34(1), 61-76 (2012). doi:10.1177/0739986311430084.

Ross, L. E. (2014). Continuing the war against domestic violence (2nd ed.). Boca Raton: CRC Press.

Scull, S., \& Woolcock, G. (2005). Problem gambling in non-English speaking background communities in Queensland, Australia: a qualitative exploration. International Gambling Studies, 5(1), $29-44$ (2005). doi:10.1080/14459790500097939.

Shankar, J., Das, G., \& Atwal, S. (2013). Challenging cultural discourses and beliefs that perpetuate domestic violence in South Asian communities: a discourse analysis. Journal of International Women's Studies, 14(1), 248-262.

Suomi, A., Jackson, A. C., Dowling, N. A., Lavis, T., Patford, J., Thomas, S. A., et al. (2013). Problem gambling and family violence: family member reports of prevalence, family impacts and family coping. Asian journal of Gambling Issues and Public Health, 3(13), 1-15.

Taft, A., Hegarty, K., \& Flood, M. (2001). Are men and women equally violent to intimate partners? Measuring Risk, 25(6), 498-500.

Tata, S. P., \& Leong, F. T. L. (1994). Individualism—collectivism, social-network orientation, and acculturation as predictors of attitudes towards seeking professional psychological help among Chinese Americans. Journal of Counselling Psychology, 41(3), 280-287 (1994). doi:10.1037/0022-0167.41.3.280.

Thomas, S., \& Yamine, R. (2000). The impact of gaming on specific cultural groups: A report to the Victorian Casino and Gaming Authority. Melbourne: Victorian Casino and Gaming Authority and Cultural Partners Australia Consortium. http://assets.justice.vic.gov.au/vcglr/resources/b1 bc0f57-c5d5-46f1-9e22-70050c99c86f/impactofgamingonspecificculturalgroups.pdf. Accessed 5 Jan 2015.

Tjaden, P., \& Thoennes, N. (2000). Extent, nature, and consequences of intimate partner violence: findings from the National Violence Against Women Survey. Washington, DC: U.S. Department of Justice Office of Justice Programs.

Tran, D. (1999). Asian gambling, family losses: a study of gambling related violence in the Vietnamese community. Richmond: Jesuit Social Services.

Trevorrow, K., \& Moore, S. (1998). The association between loneliness, social isolation and women's electronic gaming machine gambling. Journal of Gambling Studies, 14(3), 263-284.

Tse, S. (2007). Family violence in Asian communities, combining research and community development. Social Policy Journal of New Zealand/Te Puna Whakaaro, 31, 170-194. https://www.msd.govt.nz/about-msd-and-our-work/publications-resources/journals-and-magazines/social-policy-journal/spj31/31-family-violence-in-asian-communitiespages170-194.html. Accessed 20 Dec 2015.

United Nations (2010). "Keeping silent is dying". Results from the national study on domestic violence against women in Viet Nam. United Nations and General Statistics Office, Ministry of Planning and Investment. http://asiapacific. unwomen.org/en/digital-library/publications/2015/05/results-from-the-national-study-on-domestic-violenceagainst-women-in-viet-nam\#view. Accessed 15 June 2015.

Victorian Casino and Gambling Authority (VCGA) (1999). Seventh survey of community gambling patterns and perceptions. Melbourne. http://www.gamblingresearch.org/content/seventh-survey-community-gambling-patterns-andperceptions. Accessed 5 Jan 2015.

Whaley, R. B. (2001). The paradoxical relationship between gender inequality and rape: toward a refined theory. Gender \& Society, 15, 531-555.

Williams, R. J., Volberg, R. A., \& Stevens, R. M. G. (2012). The population prevalence of problem gambling: Methodological influences, standardized rates, jurisdictional differences, and worldwide trends. Report prepared for the Ontario Problem Gambling Research Centre and the Ontario Ministry of Health and Long Term Care. https://www.uleth.ca/ dspace/handle/10133/3068. Accessed 12 Dec 2015.

World Health Organisation (WHO) (2005). WHO multi-country study on women's health and domestic violence against women. Geneva.

Xu, X., Zhu, F., O'Campo, P., Koenig, M. A., Mock, V., \& Campbell, J. (2005). Prevalence of and risk factors for intimate partner violence in China. American Journal of Public Health, 95(1), 78-85.

Yick, A. G. (2000). Predictors of physical spousal/intimate violence in Chinese American families. Journal of Family Violence, 15(3), 249-267.

Yick, A. G., \& Agbayani-Siewiert, P. (1997). Perceptions of domestic violence in a Chinese American community. Journal of Interpersonal Violence, 12(6), 832-846.

Yllo, K. A. (1983). Sexual equality and violence against wives in American states. Journal of Comparative Family Studies, 14, 67-86.

Yoshihama, M. (1999). Domestic violence against women of Japanese descent in Los Angeles: two methods of estimating prevalence. Violence Against Women, 5(8), 869-897 (1999). doi:10.1177/10778019922181536.

Yoshihama, M. (2000). Reinterpreting strength and safety in a socio-cultural context: dynamics of domestic violence and experiences of women of Japanese descent. Children and Youth Services Review, 22(3/4), $207-229$.

Yoshihama, M. (2002). Battered women's coping strategies and psychological distress: differences by immigration status. American Journal of Community Psychology, 30(3), 429-452 (2002). doi:10.1023/A:1015393204820.

Yoshihama, M., \& Dabby, C. (2009). Domestic violence in Asian, native Hawaiian and Pacific Islander homes. Asian and Pacific Islander Institute on Domestic Violence, a Project of Tides Center. http://www.apiidv.org/files/Facts.StatsAPIIDV-2012.pdf. Accessed 5 Jan 2015.

Yoshioka, M. R., \& Dang, Q. (2000). Asian family violence report: a study of the Cambodian, Chinese, Korean, South Asian, and Vietnamese communities in Massachusetts. Boston: Asian Task Force Against Domestic Violence. http://www. atask.org/site/publications/family-violence-report.html. Accessed 20 Dec 2014.

Yoshioka, M. R., Dinoia, J., \& Ullah, K. (2001). Attitudes toward marital violence: an examination of four Asian communities. Violence Against Women, 7(8), 900-926. 